\title{
LA CONCIENCIA SEMIÓTICA DE LOS DOCENTES DE MATEMÁTICA EN LA CONSTRUCCIÓN COGNITIVA DE LOS CONJUNTOS INFINITOS
}

\author{
Héctor Mauricio Becerra Galindo \\ hemabe2@yahoo.es \\ https://orcid.org/0000-0002-5477-4680 \\ Universidad Distrital Francisco José de Caldas; Colombia
}

Recibido: 2020-06-18; Aceptado: 2020-11-25

"No hay noesis sin semiosis"

Raymond Duval (1995/1999)

\begin{abstract}
Resumen
En este artículo se presentan algunos resultados de la tesis doctoral sobre las problemáticas semióticas en las representaciones de los conjuntos infinitos en la práctica docente, que surge de los procesos de enseñanza y aprendizaje de los conjuntos infinitos de números, donde se evidencian dificultades en los estudiantes respecto a su construcción cognitiva. En esta investigación, se centra la atención especialmente en la caracterización de las manifestaciones de "conciencia semiótica" de los docentes sobre las representaciones de los conjuntos infinitos, a partir de su reflexión sobre las producciones y comentarios de sus estudiantes.

Palabras clave: Representación semiótica, conciencia semiótica, conjuntos infinitos, problemas semióticos.

\section{THE SEMIOTIC CONSCIOUSNESS OF TEACHERS OF MATHEMATICS IN THE COGNITIVE CONSTRUCTION OF INFINITE SETS}

\begin{abstract}
This article presents some results of the doctoral thesis on the semiotic problems in the representations of infinite sets in teaching practice, which arises from the teaching and learning processes of infinite sets of numbers, where difficulties are evident in students regarding its cognitive construction. This research is especially focus on the characterization of the teachers' "semiotic awareness" manifestations of the representations of the infinite sets, based on their reflection on the productions and comments of their students.

Keywords: Semiotic representation, semiotic consciousness, infinite sets, semiotic problems.

\section{A CONSCIÊCIA SEMIOTICA DE PROFESSORES DE MATEMÁTICA NA CONSTRUÇÃ COGNITIVA DE CONJUNTOS INFINITOS}

\section{Resumo}

Este artigo apresenta alguns resultados da tese de doutorado sobre os problemas semióticos nas representações de conjuntos infinitos na prática de ensino, que decorre dos processos de ensino e aprendizagem de conjuntos infinitos de números, onde as dificuldades são evidentes nos alunos em relação à sua construção cognitiva. Nesta pesquisa, o foco é especialmente na caracterização das manifestações de "consciência semiótica" pela professores. Nesta pesquisa,
\end{abstract}


o foco é especialmente na caracterização das manifestações de "consciência semiótica" dos professores das representações dos conjuntos infinitos, com base em sua reflexão sobre as produções e comentários de seus alunos.

Palavras- chave: Representação semiótica, consciência semiótica, conjuntos infinitos, problemas semióticos.

\section{Introducción}

En la línea de investigación relativa a la didáctica del infinito y específicamente del aprendizaje de los conjuntos infinitos, que empezó con los trabajos de investigación realizados por Duval en 1983 sobre L'obstacle du dédoublement des objects mathématiques, donde se analiza la dificultad que tienen los estudiantes para aceptar la correspondencia biunívoca llamada “de Galileo", entre los números naturales (Duval (1983) habla en este documento de los números enteros, pero se refiere a los números naturales) y el subconjunto de los números naturales cuadrados perfectos; se concluye que el desdoblamiento ${ }^{1}$ genera un obstáculo de aprendizaje, ya que los estudiantes ven el objeto de forma unívoca y no con dos caras, o pueden ver dos objetos que se interpretan de dos formas diferentes; por ejemplo, los estudiantes ven el nueve como "número natural" y no como "un cuadrado de un número natural", por lo tanto, los estudiantes encuentran un obstáculo en establecer la correspondencia biunívoca entre los números naturales y el subconjunto de los números cuadrados perfectos.

En julio de 1996 el Congreso Internacional de Educación Matemática (ICME) le dio la responsabilidad a Bruno D'Amore de chief organizer de un grupo temático, el XIV: El infinito, en el VIII ICME de Sevilla, con Raymond Duval como colaborador. En la consiguiente investigación realizada por D'Amore, se redactó “una bibliografía de más de 300 títulos, con la contribución de muchos investigadores de todo el mundo" (D'Amore, 2011, p. 23).

A partir de la bibliografía, D'Amore (1996) redacta un panorama razonado de tales investigaciones clasificadas así:

1. Falta de aceptación, por parte del estudiante, de las diversas cardinalidades transfinitas.

2. Los procesos mentales y las convicciones intuitivas llevan a los estudiantes a pensar, que en un segmento largo existan más puntos que en un segmento más corto.

\footnotetext{
${ }^{1}$ Duval (1983) deja establecido que el desdoblamiento permite distinguir el mismo objeto desde sus dos caras; por ejemplo, el número nueve se puede ver con la primera cara como un "número natural" y con la segunda cara como "un cuadrado de un número natural".
} 
3. Las aceptaciones intuitivas (misconcepciones) de aplastamiento (la convicción que todos los conjuntos infinitos tienen la misma cardinalidad) y de dependencia (la convicción que hay más puntos en una recta que en un segmento) se hallan en contradicción, pero parece que los estudiantes no se sienten interesados por volver coherentes sus creencias.

4. La dificultad que tienen los estudiantes para aceptar la correspondencia biunívoca llamada “de Galileo" entre $\mathbb{N}$ y el (su) subconjunto de los números cuadrados (Duval, 1983), además se hace referencia a la dificultad de parte de los estudiantes en el pasaje entre diversos sistemas de representación (Duval, 1995).

5. El clásico debate filosófico de origen aristotélico sobre el infinito en sentido actual y en sentido potencial.

En estas investigaciones se enfatiza que, en el proceso de enseñanza y aprendizaje de los conjuntos infinitos, se evidencian dificultades en los estudiantes respecto a su construcción cognitiva; éstas están asociadas a la dificultad objetiva de los estudiantes frente a la temática del infinito (que constituye un obstáculo epistemológico propuesto por Brousseau, 1983) ${ }^{2}$ como se concluye en las investigaciones de Fischbein, Tirosh y Hess (1979), Duval (1983), Moreno y Waldegg (1991), Arrigo y D'Amore (1999, 2002), Tsamir (2000), Arrigo, D'Amore y Sbaragli (2011), entre otros; a la temática general de la formación de una noética frente a representaciones semióticas como es propuesto en la paradoja de Duval “[...] de una parte, el aprendizaje de los objetos matemáticos sólo puede ser un aprendizaje conceptual y, de otra parte, es sólo a través de representaciones semióticas que es posible una actividad sobre los objetos matemáticos" (Duval, 1993, p. 38; en la traducción D'Amore, 2002), y que es consolidado por Duval (1995/1999, p. 15) con su hipótesis "no hay noesis sin semiosis".

Las anteriores dificultades ya fueron tema de las investigaciones acabadas de comentar, por lo cual nuestra atención en este trabajo se dirige a documentar en varios casos empíricos la falta de "conciencia semiótica" que usan los docentes al elegir las representaciones para la construcción cognitiva de los conjuntos infinitos con sus estudiantes. Con más precisión, con "conciencia semiótica" se quiere indicar el conocimiento consciente sobre los sistemas de

\footnotetext{
2 Brousseau (1983) establece un obstáculo epistemológico como "un conocimiento estable que funciona bien en ámbitos anteriores, pero que crea problemas y errores cuando se le intenta adaptar a nuevas situaciones” (Arrigo, D'Amore, \& Sbaragli, 2011, p. 135).
} 
representaciones, que se movilizan en la actividad matemática y que es específica de la semiótica.

Estas dificultades se empiezan a evidenciar cuando los docentes generan argumentos desde lo que ven en las representaciones y no desde la coordinación de registros de representaciones semióticas que son necesarios para la conceptualización (Duval, 1993) de los conjuntos infinitos; por ejemplo, los docentes eligen para su cátedra la siguiente representación gráfica (figura 1) que es habitual en los libros de texto.

Figura 1. Representación gráfica de $\mathbb{N}$ y $\mathbb{Z}$.

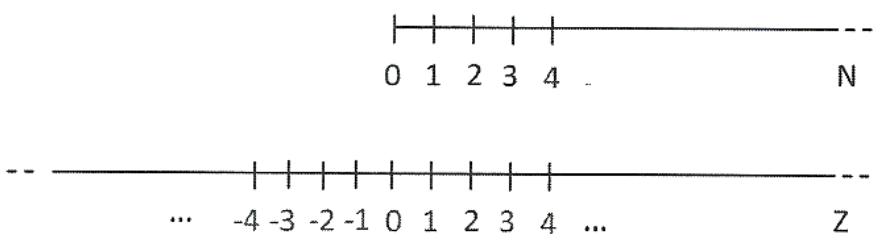

Fuente: Arrigo, D'Amore, \& Sbaragli (2011, p. 222).

Esta representación gráfica lleva a los docentes (se codifica con la letra C y F) a proporcionar los siguientes argumentos a las preguntas realizadas por los investigadores (codificado como Inv).

Inv: [...] ¿Tú crees que los elementos que forman el conjunto de los enteros son: más, menos o el mismo número de los elementos que tiene el conjunto de los naturales?

C: Obviamente son más, están además todos los negativos.

Inv: ¿Cómo representarías estos conjuntos numéricos a tus estudiantes?

$C$ : Los relativos [enteros] los pondría en la recta de los números y los naturales en cambio deben estar en la línea de los números. [...]

Inv: ¿Esto lo presentas en clase?

C: Por supuesto que digo que los números negativos siempre deben estar siempre antes de los positivos. (Arrigo, D'Amore, \& Sbaragli, 2011, p. 208)

En este caso la representación gráfica lleva a pensar, tanto a los docentes como a los estudiantes, que el número de enteros es “el doble” de los números naturales, en otras palabras, que el conjunto de los números enteros tiene más elementos que el conjunto de los números naturales.

Evidenciemos otro argumento de otro docente al dar respuesta a la pregunta 
Inv: ¿Cuántos son los números naturales: $0,1,2,3, \ldots$ ?

F: Los números naturales son infinitos, ya que un conjunto es infinito si está conformado por infinitos elementos y $0,1,2,3, \ldots$ son infinitos. (Arrigo et al., 2011, p. 215)

Este argumento no presenta una construcción cognitiva del objeto conjunto infinito, ya que: "Un conjunto $\mathrm{S}$ se denomina infinito sí y solo sí se puede poner en correspondencia biunívoca con una de sus partes propias" (Arrigo et al., 2011, p. 97). Lo que se evidencia en el anterior argumento es la interpretación que el docente $\mathrm{F}$ le está dando a los puntos suspensivos en la representación $0,1,2,3, \ldots$ como los infinitos elementos que conforman el conjunto; en este caso el docente $\mathrm{F}$ se refiere al infinito en potencia.

En estos ejemplos se muestra en cierta medida la falta de "conciencia semiótica" que tienen los docentes en la construcción cognitiva y en la enseñanza de los conjuntos infinitos; pero esta no es la única causa; los docentes, para los procesos de enseñanza - aprendizaje de los objetos matemáticos, recurren a los libros de texto de matemática como referentes para su planeación y diseño de actividades.

Por ejemplo, para la construcción cognitiva de los números reales se proponen en los libros de textos de secundaria (grados octavo $\left(8^{0}\right)$ y once $\left(11^{0}\right)$ ) las siguientes definiciones.

\section{Definición libro para grado $8^{0}$}

Los números naturales $\mathbb{N}$, los enteros $\mathbb{Z}$ y los racionales $\mathbb{Q}$, conforman, junto con los irracionales $\mathbb{I}$., el conjunto de los números reales $\mathbb{R}$. Para entender cómo se relacionan los conjuntos de números mencionados, observemos el siguiente esquema:

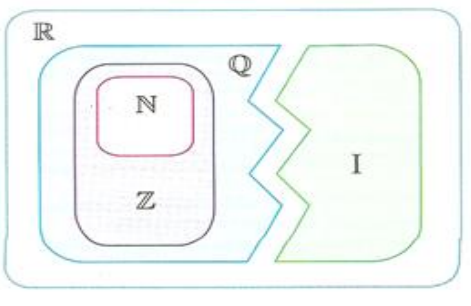

. (Dueñas, Garavito, \& Lara, 2007, p. 48)

En esta definición, se puede observar un problema en la representación semiótica (Becerra Galindo, 2017, 2018; Becerra Galindo \& Font, 2019) que generan la contradicción entre el registro de la lengua natural y la representación auxiliar; la cual no está representando correctamente el objeto matemático números reales, en otras palabras no se está asociando al mismo objeto inaccesible (Duval, 1995/1999, 2006, 2017; Duval \& Sáenz-Ludlow, 2016); en 
este caso no existe una coordinación de registros (Duval, 1995/1999, 2004, 2006, 2017; Duval \& Sáenz-Ludlow, 2016).

\section{Definición libro para grado $11^{0}$}

El conjunto de los números naturales lo representamos con $\mathbb{N}$ y está formado por los números que se utilizan para contar es decir, $\mathbb{N}=\{1,2,3, \ldots\}$. A partir de los números naturales es posible construir los números enteros $\mathbb{Z}$ agregando el 0 y los negativos de los números naturales. De esta forma obtenemos $\mathbb{Z}=\{\ldots,-3,-2,-1,0,1,2,3, \ldots\}$.

Es posible representar estos números en la recta numérica como mиеstra la figura

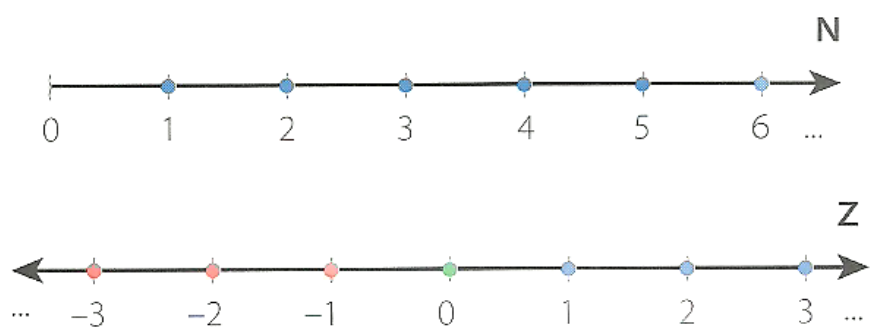

Alformar todos los posibles cocientes entre números enteros obtenemos el conjunto $\mathbb{Q}$. Entonces $\mathbb{Q}=\left\{\frac{a}{b}: a, b \in \mathbb{Z}, b \neq 0\right\}$. Los números racionales se caracterizan porque su expresión decimal es finita o infinita periódica. Aquellos números cuya expresión decimal es infinita no periódica forman el conjunto de los números irracionales II. Algunos ejemplos importantes de los números irracionales son: $\pi \approx 3,14159 \ldots$, $e \approx 2,71828 \ldots$

"Los números reales son el conjunto formado por la unión de los números racionales y los irracionales. $\mathbb{R}=\mathbb{Q} \cup \mathbb{I}$. ” (Moreno, Roldán, \& Romero, 2011, p. 12)

En el registro de representación simbólica solo se presenta por parte de los autores del libro de texto el orden "natural" de los números naturales y de los números enteros, lo que lleva a docentes y estudiantes a pensar que solo hay estas representaciones posibles y correctas, como lo afirma un docente "estos números tienen que ordenarse siempre así" (Arrigo et al., 2011, p. 209); además, puede ser sustentado desde la convicción de algunos docentes cuando observan el registro gráfico y establecen que $\mathbb{N}$ tiene un verso hacia el infinito (positivo) con un orden "natural", y $\mathbb{Z}$ aunque tiene dos versos hacia el infinito (positivo y negativo) sigue presentando un orden "natural", argumento que no es válido y que depende de cómo eliges el orden y de cómo lo representas. Y entonces el problema verdadero en estos casos es la representación semiótica de los conjuntos infinitos. 
Para afrontar estas dificultades es necesario: 1) Que el docente reflexione sobre la importancia que tiene la elección de representaciones de los conjuntos infinitos en su construcción cognitiva, 2) que se dé cuenta que esta elección no es univoca ni neutra y 3) que puede ser una causa de la falta de construcción cognitiva por parte de sus estudiantes.

Por lo tanto, surge la siguiente pregunta de investigación: ¿Cuáles manifestaciones de la conciencia semiótica se producen en el docente cuando se le problematiza su elección de representaciones semióticas en el proceso de enseñanza - aprendizaje de los conjuntos infinitos?

Dicha pregunta se concreta en diferentes objetivos específicos, siendo uno de ellos: Caracterizar las manifestaciones de conciencia semiótica de los docentes sobre las representaciones de los conjuntos infinitos, a partir de su reflexión sobre las producciones y comentarios de sus estudiantes.

\section{Marco teórico}

Las referencias que se abordan para este artículo están relacionadas especialmente con los elementos semióticos-cognitivos propuestos en la teoría Raymon Duval (1993, 1995/1999, 1996, 2004, 2006, 2008, 2016, 2017).

\section{Representaciones semióticas}

En la psicología cognitiva, la noción de representación juega un papel importante con respecto a la adquisición y el tratamiento del conocimiento de un individuo (Pino-Fan, Guzmán, Duval, \& Font, 2015). En palabras de Duval (1995/1999, 2004, 2016) una representación es "algo que se pone en lugar de otro algo" (Duval \& Sáenz-Ludlow, 2016, p. 62).

La estructura que propone Duval (2008) de una representación semiótica es:

\{\{contenido de la representación, registro semiótico representado\}, objeto representado\}.

Los registros que se movilizan en matemáticas son cuatro: discursivos, no discursivos, multifuncionales y monofuncionales. Duval (2004) define los registros discursivos como los que permiten describir, inferir, razonar, calcular. Los registros no discursivos como los que permiten visualizar lo que nunca es dado de manera visible. Los registros multifuncionales como los que son utilizados en todos los dominios de la vida cultural y social. Los registros monofuncionales como registros derivados, que son especializados en un solo tratamiento. En la figura 2, se presenta la clasificación de los registros que son movilizados en matemáticas. 
Figura 2. Clasificación de los diferentes tipos de registros movilizados en matemáticas

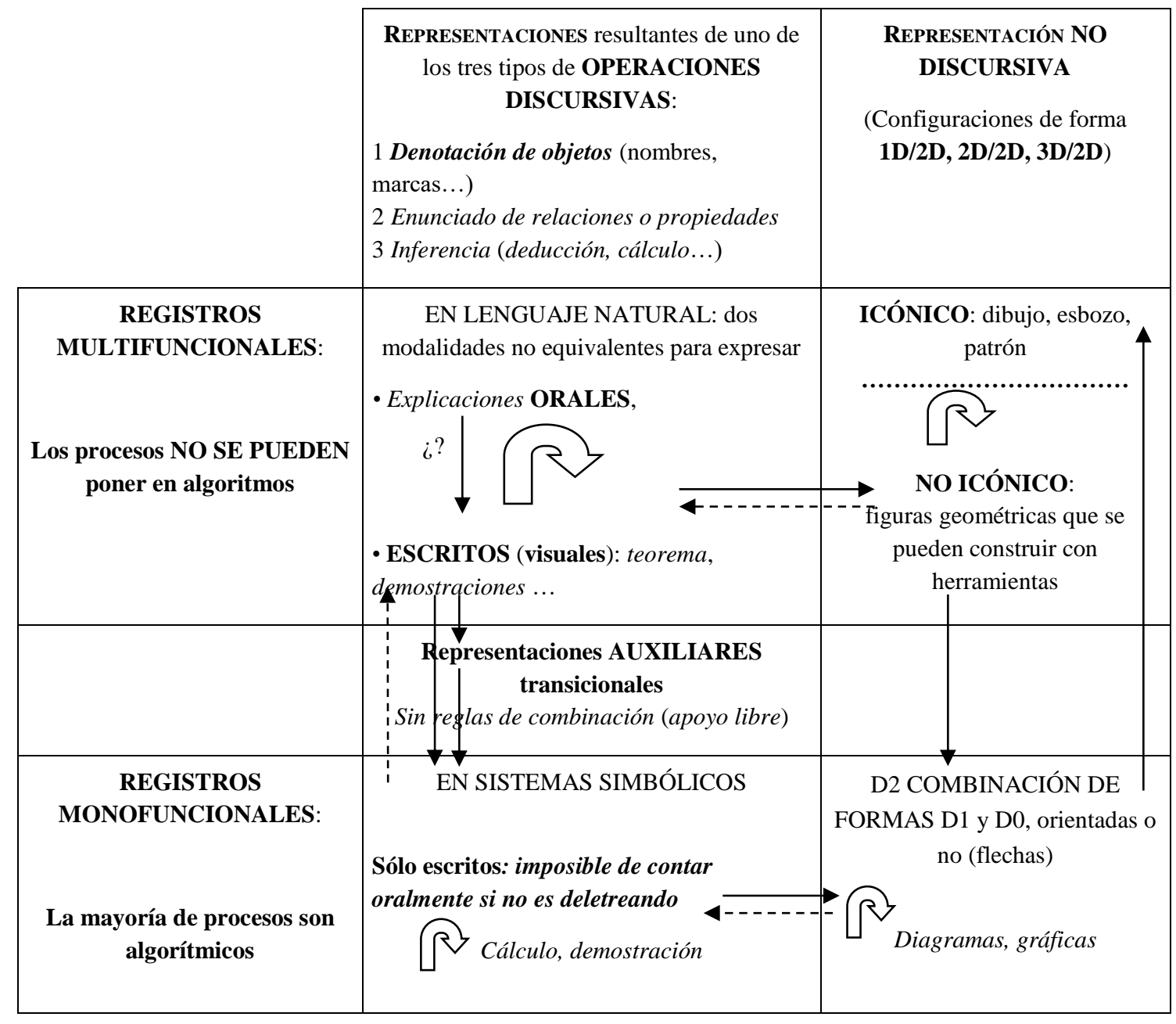

Fuente: Duval \& Sáenz-Ludlow (2016, p. 71).

Es necesario aclarar que existen unas representaciones auxiliares que no dependen del registro semiótico y son utilizadas en matemáticas, como el material (por ejemplo, el manipulativo como: el ábaco, las regletas de Cuisenaire, bloques lógicos, etc.), los ejemplos, las ilustraciones, la organización (las tablas), etc. (Duval, 2004).

En los procesos de pensamiento que están involucrados con la actividad matemática, es necesario enfocarse en el nivel de los sistemas semióticos y no en la representación particular producida (Duval \& Sáenz-Ludlow, 2016), ya que es en este nivel donde se captura la importancia de la representación semiótica en la matemática.

Los dos tipos de transformaciones que se dan en la representación semiótica son: los tratamientos y las conversiones. 
Los tratamientos son transformaciones en el mismo registro; por ejemplo: a) $1 / 2 \rightarrow 0,5$, se pasa de un registro de escritura fraccionaria a un registro de escritura decimal y b) $x-y+3$ $=0$ a $y=x+3$, la representación cambia, pero el registro de la escritura algebraica no (Iori, 2014), en estos dos ejemplos se sigue conservando el registro monofuncional y registro discursivo.

Las conversiones son transformaciones de representaciones que consiste en cambiar un registro sin cambiar los objetos denotados; por ejemplo, pasar del registro de la lengua natural al registro pictográfico, así:

\section{Un medio $\rightarrow \square$ (Fandiño Pinilla, 2010, p. 37).}

Para Duval (1995/1999) el estudio de la actividad de conversión permite comprender la estrecha relación entre "noesis" y "semiosis", que es esencial en el aprendizaje de los estudiantes.

Esta última transformación es la raíz de "los problemas que muchos estudiantes tienen con el pensamiento matemático [... por su...] complejidad cognitiva [...y por el...] cambio de representación” (Duval \& Sáenz-Ludlow, 2016, p. 85).

\section{Metodología}

Esta investigación presenta un análisis de las representaciones de los conjuntos numéricos elegidas en su cátedra por los docentes en la construcción cognitiva de los conjuntos infinitos por sus estudiantes. Para establecer las manifestaciones de conciencia semiótica de los docentes, se tuvo en cuenta la siguiente opción metodológica:

1) Se observaron y se grabaron las clases de los docentes, evidenciado la elección de las representaciones semióticas de los conjuntos numéricos utilizadas por el docente para la construcción cognitiva de los conjuntos infinitos;

2) se tomaron las frases, fragmentos de video y las hojas respuesta de sus estudiantes, que evidenciaron la no construcción del objeto matemático conjunto infinito a partir de la elección de representaciones semióticas de los conjuntos numéricos propuestas por el docente $\mathrm{y}$ 
3) se formularon preguntas al docente relacionadas con la elección de representaciones semióticas para alcanzar la construcción cognitiva de los conjuntos infinitos por parte de sus estudiantes; se le mostraron las respuestas de sus estudiantes, las cuales evidenciaron la no construcción cognitiva del objeto matemático conjunto infinito (fragmentos de video, frases y hojas de respuestas de sus estudiantes). En la respuesta (datos) dada por el docente se evidenciaron las manifestaciones de su conciencia semiótica sobre la elección de las representaciones semiótica de los conjuntos numéricos utilizados para favorecer la construcción cognitiva de los conjuntos infinitos.

\section{Análisis de los datos}

A continuación, se muestra un análisis de las problemáticas semióticas de las representaciones de los conjuntos numéricos elegidas en su cátedra por los docentes $\mathrm{D}_{11}{ }^{3}$ (D: Docente; 11: Colegio República Bolivariana de Venezuela IED) y $\mathrm{D}_{21}$ (D: Docente; 21: Colegio Tomás Cipriano de Mosquera IED), para la construcción cognitiva de los conjuntos infinitos. Se analizarán especialmente la representación auxiliar de conjunto y la representación gráfica.

\section{Representación auxiliar de conjunto}

En este apartado se muestra la representación auxiliar de conjunto elegida y explicada por los docentes $\mathrm{D}_{11}$ y $\mathrm{D}_{21}$ en cada una de sus cátedras, y que es construida por sus estudiantes.

Inicialmente, el investigador muestra al docente $\mathrm{D}_{11}$ un segmento de video de la entrevista hecha a sus estudiantes sobre el tipo de representación que ellos realizan de los conjuntos [150] (este código significa la transcripción escrita de la entrevista al docente en el párrafo 150) y se le realizan las siguientes preguntas en la entrevista:

[...] [151]: ¿Está bien lo realizado por la estudiante $\mathrm{E}_{11}$ ? (figura 3), el docente $\mathrm{D}_{11}$ responde [152]: "Pues, se ven algunos errores, si claro, el conjunto estaría bien, el error o dificultad es que pone al mismo nivel los números racionales e irracionales, o sea, en este caso no se dio cuenta de la diferencia entre los números irracionales y los números racionales”, se pregunta nuevamente [153]: ¿No es claro que los números racionales son

\footnotetext{
${ }^{3}$ De aquí en adelante se utilizará este tipo de codificación.
} 
diferentes a los números irracionales en los conjuntos?, el docente $\mathrm{D}_{11}$ responde [154]: "Exacto".

Figura 3. Representación auxiliar $\mathrm{E}_{11}$

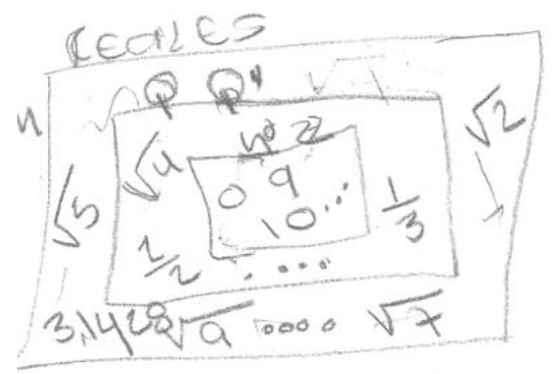

Se continúa con la entrevista y se pregunta al docente $\mathrm{D}_{11}$ [155]: ¿Se había trabajado en clase esta representación de conjuntos?, el docente $\mathrm{D}_{11}$ responde [156]: "Si eso se trabaja en clase, pero entonces, los números irracionales se ubican por fuera de los números racionales" (figura 4), nuevamente se pregunta [157]: ¿O sea que la representación no es clara?, el docente $D_{11}$ responde [158]: "Exacto, es decir de todas maneras, el complemento de los números racionales no está mostrado en la representación [muestra la representación realizada por la estudiante (figura 3)], no diferenciaron $\mathbb{Q}$ y $\mathbb{Q}$ ”.

Figura 4. Representación auxiliar de $\mathrm{D}_{11}$ : Conjunto de los números irracionales

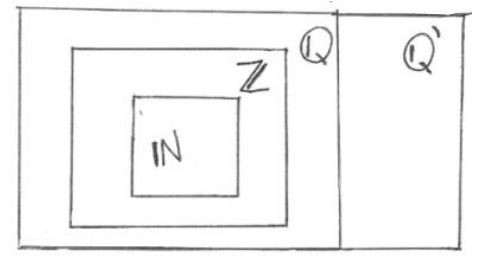

En esta parte de la entrevista, el docente $\mathrm{D}_{11}$ reconoce que la representación auxiliar de $E_{11}$ (figura 3) presenta errores y dificultades, porque pone en el mismo conjunto los números racionales e irracionales, $\mathrm{E}_{11}$ no se dio cuenta de la diferencia entre el conjunto de los números irracionales $(\mathbb{Q})$ y de los números racionales $\left(\mathbb{Q}^{\prime}\right)$. A pesar que esta representación es trabajada en la cátedra del docente $\mathrm{D}_{11}$, no es clara para los estudiantes la representación auxiliar de conjunto.

Con respecto a la entrevista de la docente $\mathrm{D}_{21}$, se propone el análisis de la representación de $\mathrm{E}_{24}$ (figura 5), donde se explica que $\mathrm{E}_{24}$ pone los números irracionales (II) a parte de los números racionales $(\mathbb{Q})$ y encierra con un cuadrado los números reales $(\mathbb{R})$; se pregunta al 
estudiante: Si se pone hipotéticamente un punto en este espacio entre números racionales e irracionales (figura 5), y el punto no pertenece a ninguno de los dos ¿Qué número es?, ¿a qué conjunto pertenece?; el estudiante no pudo responder.

Figura 5. Representación auxiliar $\mathrm{E}_{24}$

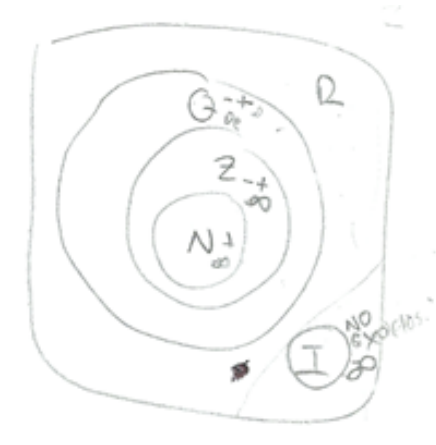

Luego, se explica a la docente $\mathrm{D}_{21}$ en la entrevista:

[...] la representación de $\mathrm{E}_{24}$ [243] tiene la problemática que poniendo ese punto en ese espacio no haría parte de los números reales, la docente $\mathrm{D}_{21}$ afirma que ese punto (número) hipotético [244]: "Es real, lo que pasa es y la discusión es, si es racional o si es irracional que no lo es, pero si es real, viendo el real como el padre de todos los números, es el macro sistema de todos los conjuntos numéricos". Se vuelve a preguntar a la docente $\mathrm{D}_{21}$, [245]: Pero si el punto que hipotéticamente es un número y no es irracional, ni racional ¿Qué sería?, la docente $\mathrm{D}_{21}$ responde [246]: "No sé, ya me confundí". En otro apartado de la entrevista la docente $\mathrm{D}_{21}$ afirma [242]: "La representación que realicé en la clase (Figura 6) y que más se acercó fue ésta de $\mathrm{E}_{24}$ (figura 5), sólo que debe colocar la $\mathbb{R}$ afuera".

Figura 6. Representación auxiliar de $\mathrm{D}_{21}$ : Conjunto de los números reales.

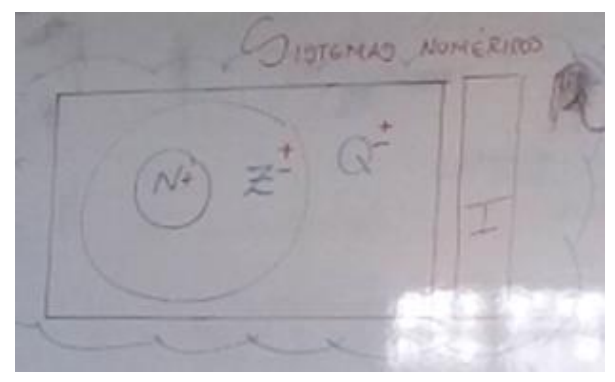


En este caso específico de la elección de la representación auxiliar de los conjuntos numéricos por parte de la docente $\mathrm{D}_{21}$ (figuras 6), se presenta una problemática semiótica desde el "saber matemático", ya que al elegir la representación auxiliar del libro de texto Aciertos matemáticos $8, \mathrm{D}_{21}$ no realiza un análisis crítico a nivel semiótico y sólo opta por tomar la transposición didáctica elegida por el autor del libro asimilándose a este (Arrigo et al., 2011). La problemática semiótica que se evidencia es:

[...] si se ubica una representación hipotética de dos números [en la figura 5 es el punto negro] en la representación auxiliar donde está la letra $\mathbb{R}$, se puede establecer que en esos lugares el números no pertenecen a $\mathbb{Q}$, ni a II, entonces surge la siguiente pregunta: ¿Qué tipo de números se representarían en este lugar?; lo que se tiene en este ejemplo es un problema con la representación auxiliar, la cual no está representando correctamente el objeto matemático de los números reales. (Becerra Galindo, 2017, p. 196, 2018, p. 6; Becerra Galindo \& Font, 2019. p. 537)

Es evidente que la docente $\mathrm{D}_{21}$ no cuenta con los elementos necesarios desde la semiótica para realizar un análisis crítico en la elección de la representación auxiliar, lo que lleva a generar dificultades en el aprendizaje de sus estudiantes sobre la conceptualización del conjunto de los números reales y los conjuntos infinitos.

\section{Representación gráfica}

En este apartado se muestra la representación gráfica elegida y explicada por los docentes $\mathrm{D}_{11}$ y $\mathrm{D}_{21}$ en cada una de sus cátedras, y que construyeron sus estudiantes.

El docente $\mathrm{D}_{11}$ reconoce que una de las representaciones de los números reales es la recta, cuando se le pregunta: ¿Una representación de los números reales sería la recta numérica?, el docente $\mathrm{D}_{11}$ responde [32]: "Exactamente”. También se le consulta sobre la conceptualización de los números racionales a partir de la representación gráfica de la recta así:

Se pregunta por las representaciones simbólicas realizadas por el docente en la representación gráfica (figura 7), se pregunta [39]: [Se señalan los números racionales $\left(\frac{5}{4}\right)$ y $\left.\left(\frac{6}{4}\right)\right]$ ¿Son fracciones?, el docente $\mathrm{D}_{11}$ responde [40]: “Eh, los números racionales son todos aquellos que se pueden expresar como fracción, sí, entonces se está con los números racionales, con los números de forma racional o de forma decimal, ellos [los estudiantes] hicieron un trabajo de conversión de decimal a fracción y de fracción a 
decimal". El investigador señala los dos números racionales $\left[\left(\frac{5}{4}\right)\right.$ y $\left.\left(\frac{6}{4}\right)\right]$ y pregunta [41]: ¿En este caso no sería fracción sino número racional?, el docente $\mathrm{D}_{11}$ responde [42]: “Exacto, la fracción es una forma de representación del número racional, escribir un número como fracción es una forma de representar el número racional”.

Figura 7. Representación gráfica de los números racionales de $\mathrm{D}_{11}$

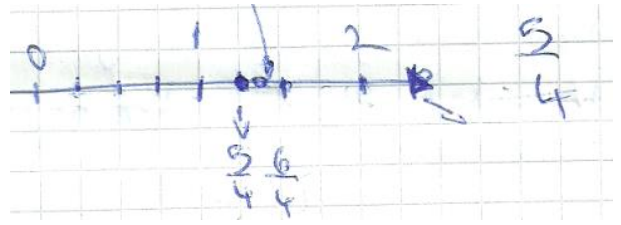

En este caso el docente $\mathrm{D}_{11}$ establece que la fracción es una forma de representación del número racional, ya que en la cátedra propone que $\left(\frac{5}{4}\right)$ y $\left(\frac{6}{4}\right)$ son fracciones y los estudiantes reconocen, interpretan y comprenden que los números racionales negativos son fracciones; además, se generan ciertas dificultades en ubicar los números racionales negativos como en el caso de $E_{11}$, quien ubica $\frac{-3}{5}$ entre -4 y -3 , discusión que se propone en la siguiente parte de la entrevista.

[...] el investigador señala la recta antes de cero (0) de la representación realizada por $\mathrm{E}_{11}$ (figura 8) y $\mathrm{E}_{13}$ (figura 9), y realiza la siguiente pregunta al docente $\mathrm{D}_{11}$ [43]: ¿Qué pasa con los números que son menores de cero (0)?, el docente $\mathrm{D}_{11}$ responde [44]: "En el caso de los negativos exactamente es lo mismo, por ejemplo, menos tres cuartos $\left(\frac{-3}{4}\right)$, se divide la unidad en cuatro partes y se toman tres partes. Otro ejemplo, menos siete cuartos $\left(\frac{-7}{4}\right)$, se toma la segunda unidad, se divide y se ubica $\left(\frac{-7}{4}\right)$, en el caso de querer representarlo como fracciones, sí” (figura 10); se pregunta nuevamente [45]: ¿Menos tres cuartos $\left(\frac{-3}{4}\right)$ es una fracción?, el docente $D_{11}$ afirma [46]: "Menos tres cuartos $\left(\frac{-3}{4}\right)$ es una fracción, no, es un número racional, pero lo estoy representando a través de las fracciones", vuelve y se pregunta al docente $D_{11}$ [47]: ¿Menos tres cuartos $\left(\frac{-3}{4}\right)$ no es una fracción?, el docente $\mathrm{D}_{11}$ responde [48]: "El conjunto al que pertenece menos tres cuartos $\left(\frac{-3}{4}\right)$ es al conjunto de los números racionales". 
Figura 8. Representación gráfica $\mathrm{E}_{11}$

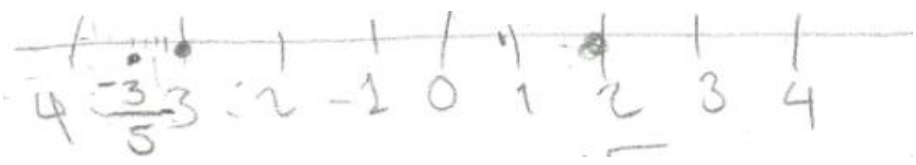

Figura 9. Representación gráfica $\mathrm{E}_{13}$

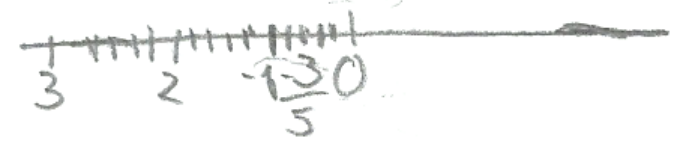

Figura 10. Representación gráfica de los números racionales de $\mathrm{D}_{11}$

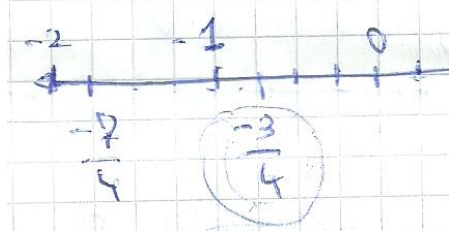

Siguiendo con la entrevista se pregunta nuevamente [49]: ¿Menos tres cuartos $\left(\frac{-3}{4}\right)$ es una fracción?, el docente $\mathrm{D}_{11}$ responde [50]: "Es la forma como se expresa el número racional”, se pregunta [51]: ¿No es una fracción?, el docente $\mathrm{D}_{11}$ responde [52]: "Yo hubiera podido escribir menos cero coma setenta y cinco (-0.75)", se pregunta [53]: ¿Qué es menos cero coma setenta y cinco (-0.75)?, el docente $\mathrm{D}_{11}$ afirma [54]: "Es un número racional y a la vez es un número real”, se pregunta [55]: ¿Por qué?, ¿cómo sería la definición de un número racional?, el docente $\mathrm{D}_{11}$ establece la siguiente definición [56]: "Son todos aquellos números que se pueden expresar como una fracción, este número 0.75 se puede expresar como una fracción”.

En esta parte de la entrevista, se evidencia que el docente $\mathrm{D}_{11}$ reconoce que escribir un número racional como fracción es una forma de representarlo simbólicamente, además reconoce la transformación de tratamiento, pasando, en el mismos registro semiótico, a otra representación diferente; en este caso, el docente se ubica en el registro monofuncional con la representación simbólica de una fracción y realiza la transformación de tratamiento pasando a la representación simbólica de un decimal, reconociendo ésta como un número racional.

El problema, en este caso, es que, aunque el docente tiene claridad sobre los conceptos, éstos no fueron explicados en su cátedra, sólo afirma que los números racionales se pueden 
escribir en forma de fracción, por lo tanto, los estudiantes entienden que menos tres cuartos $\left(\frac{-3}{4}\right)$ es una fracción y no un número racional, algo que no es correcto y que el docente $\mathrm{D}_{11}$ debe aclarar en su cátedra.

Con respecto a la entrevista realizada a la docente $\mathrm{D}_{21}$, se pregunta sobre el significado de ciertos elementos de la representación gráfica:

[...] [134]: Como se puede ver, algunos estudiantes ponen las flechitas en la recta, pero donde colocan los números en la recta no hay nada (figuras 11 y 12 ); la docente $\mathrm{D}_{21}$ responde [135]: "No es necesario los puntos”. Se pregunta [136]: ¿Será, que no son necesarios los puntos en los números o que sólo tenga los puntos en los números y no la flecha?; la docente $\mathrm{D}_{21}$ contesta [137]: "Yo pienso, que la flecha, los tres puntos y el símbolo del infinito como ocho acostado $(\infty)$, representan lo mismo, lo que yo estoy viendo es que el estudiante hace los tres, él $\left(\mathrm{E}_{22}\right)$ quiere reforzar que allí hay una infinidad de números tanto a la derecha de la recta como a la izquierda, y hace la flecha y los tres puntos, y el símbolo del infinito que es el ocho acostado ( $\infty)$, pero para representar lo mismo, lo que pienso es que uno es suficiente, con solo uno de ellos es suficiente". Se pregunta nuevamente [138]: ¿Sólo sería la flecha o los puntos o el símbolo de infinito?; la docente $\mathrm{D}_{21}$ responde [139]: "En este caso, con el hecho de hacer la flecha ya está indicando la infinidad".

Figura 11. Representación gráfica $\mathrm{E}_{26}$

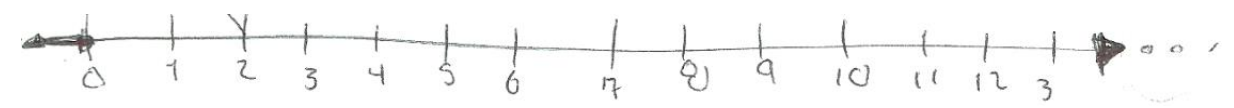

Figura 12. Representación gráfica $\mathrm{E}_{22}$.

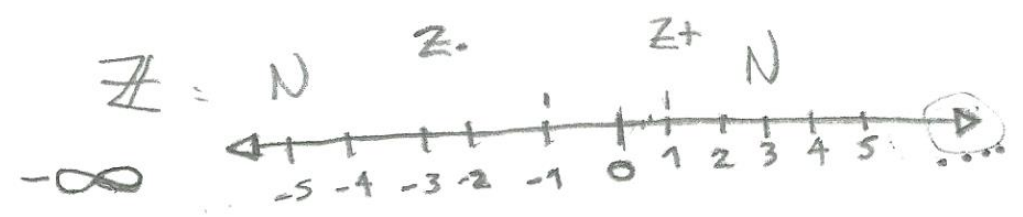

La docente $\mathrm{D}_{21}$ reconoce en la representación gráfica de la recta, que la flecha, los puntos suspensivos en los números y el símbolo de infinito $(\infty)$, están representando el infinito. En la entrevista, la docente $\mathrm{D}_{21}$ propone que no es necesario poner la flecha, los puntos suspensivos y 
el símbolo de infinito ( $\infty$ ) en la recta numérica, ya que "para representar lo mismo, lo que pienso es que uno es suficiente".

La anterior afirmación de la docente $\mathrm{D}_{21}$ generaría un problema con los estudiantes, al comprender estas representaciones del infinito en la gráfica de los textos escolares, ya que en éstos se proponen dos o los tres tipos de estas representaciones del infinito.

Posteriormente se pregunta a la docente $\mathrm{D}_{21}$ por las representaciones gráficas de los números naturales de $\mathrm{E}_{25} \mathrm{y} \mathrm{E}_{26}$, donde el primero pone los números 1, 2, 3, 4, 5, 6, y una flechita adelante y una atrás, sin poner la semirrecta (figura 13) y el segundo realiza la recta con una flechita y unos puntos suspensivos (figura 11).

El investigador pregunta [227]: ¿Qué pasa con estas representaciones?, la docente $\mathrm{D}_{21}$ responde [228]: "De pronto, le pasó lo mismo que a mí cuando empecé a realizar los números naturales y dibujé la recta numérica, pero dije, no esta parte no, porque es una semirrecta, si se habla de los números naturales, pero eso también me pasó a mí".

Figura 13. Representación gráfica $\mathrm{E}_{25}$

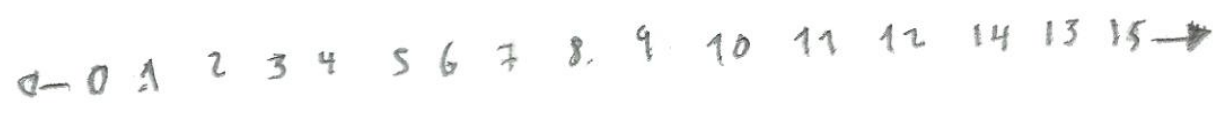

En esta parte de la entrevista, la docente $\mathrm{D}_{21}$ afirma que los estudiantes tienen el mismo problema que ella misma con la representación gráfica de los números naturales, ya que los números naturales se representan gráficamente en una semirrecta y no en la recta; en este caso, la docente empieza a crear una conciencia semiótica sobre el sistema de representación gráfico de los números naturales que tienen como modelo más natural de representación al representarlos una semirrecta y el de los números enteros, en una recta.

En otra parte de la entrevista.

[...] se hace la siguiente pregunta a la docente $\mathrm{D}_{21}[61]: ¿ \mathrm{La}$ infinidad la representa la recta, el ocho acostado y los conjuntos?, la docente $\mathrm{D}_{21}$ responde [62]: “En los conjuntos no $[\ldots]$ pues sabe que si podría representar un conjunto infinito, porque es que si se habla de $\mathbb{Z}$, se hablaría de enteros y los números enteros son un conjunto infinito; sin embargo, la infinidad es mucho más clara en la recta, pero ahora que reflexiono, pues el solo hecho 
de ver la $\mathbb{Z}$, implica una infinidad, porque se está representando un conjunto que es infinito".

En esta parte de la entrevista, la docente $\mathrm{D}_{21}$ declara que "la infinidad es mucho más clara en la recta", que es una representación de un conjunto infinito, pero define un conjunto infinito como "aquel que tiene infinitos elementos que hacen parte de él”, definición que no permite la construcción cognitiva de los conjuntos infinitos, dado que esta necesita de un infinito actual.

\section{Resultados}

Se caracterizan las siguientes manifestaciones de la conciencia semiótica de los docentes $\mathrm{D}_{11}$ y $\mathrm{D}_{21}$, así:

1. La representación auxiliar del conjunto de los números reales propuesta en la cátedra por el docente $\mathrm{D}_{11}$ (figura 4) no tiene ninguna similitud con la representación auxiliar que los estudiantes propusieron en su entrevista. El docente $\mathrm{D}_{11}$ reconoce al ver el fragmento del video y las representaciones auxiliares hechas en las hojas por los estudiantes, que en las representaciones auxiliares de $\mathrm{E}_{11}$ (figura 3) se presenta errores y dificultades, debido a que ponen en el mismo conjunto los números racionales e irracionales, $\mathrm{E}_{11} \mathrm{y}$ $\mathrm{E}_{13}$ no se dan cuenta de la diferencia entre el conjunto de los números racionales $(\mathbb{Q})$ y de los números irracionales $\left(\mathbb{Q}^{\prime}\right)$.

2. La representación auxiliar del conjunto de los números reales elegida por la docente $\mathrm{D}_{21}$ para su cátedra (figura 6) presenta una problemática semiótica (caracterizada por Becerra Galindo, 2017, 2018; Becerra Galindo \& Font, 2019), donde la docente $\mathrm{D}_{21}$ no realiza un análisis crítico a nivel semiótico y sólo opta por tomar la transposición didáctica elegida por el autor del libro asimilándose a éste (Arrigo et al., 2011).

Este problema semiótico de la representación auxiliar fue enseñado y aprendido por los estudiantes (figura 5 de $\mathrm{E}_{24}$ ), y puesto de manifiesto en el anterior análisis cuando se pregunta a los estudiantes [231]: Si se pone hipotéticamente un punto [se pone un punto negro (figura 5)] en este espacio entre los números racionales y números irracionales, ¿Qué número es?, ¿a qué conjunto pertenece?, los estudiantes no responden. Esta duda que presentan los estudiantes sobre la imagen de la representación auxiliar, los puede llevar muy rápidamente a consolidar un modelo que la docente $\mathrm{D}_{21}$ ya ha construido de 
manera inadecuada, formando en el estudiante un conflicto cognitivo (D'Amore, 1999/2006).

3. La conversión entre la representación gráfica en la recta y la representación simbólica, ya que $\mathrm{E}_{11}$ no representa gráficamente bien el número racional $\frac{-3}{5}$ (figura 8 ), esto se debe a dos hechos: El primero relacionado con que los estudiantes tienen la concepción que $\frac{-3}{5}$ es una fracción, por lo tanto, no existe una fracción negativa y el segundo relacionado con que los estudiantes no pueden representar gráficamente $\frac{-3}{5}$ en la recta, por ejemplo, E11 ubica el $\frac{-3}{5}$ entre los números -3 y -4 (figura 8 ).

Frente a esta problemática de conversión de las representaciones y la concepción de la fracción como negativo en los argumentos de los estudiantes, el docente $\mathrm{D}_{11}$ afirma [42]: "La fracción es una forma de representación del número racional” y [46]: "Menos tres cuartos $\left(\frac{-3}{4}\right)$ no es una fracción, es un número racional, pero lo estoy representando a través de las fracciones"; sin embargo, los estudiantes no entienden que menos tres cuartos $\left(\frac{-3}{4}\right)$ es un número racional, como lo reconoce el docente $\mathrm{D}_{11}$ "menos tres cuartos $\left(\frac{-3}{4}\right)$ es un número racional". Por lo tanto, el docente $\mathrm{D}_{11}$ debe planear y aclarar en su cátedra todos estos elementos semióticos y conceptuales (fracción, infinito y número racional), como lo realizó en la entrevista.

4. La docente $\mathrm{D}_{21}$ no cuenta con los elementos semióticos que le permitan establecer las diferencias de las representaciones gráficas de los números naturales (semirrecta) y los números enteros (recta). En el análisis se evidencia que tanto la docente $\mathrm{D}_{21}$ como los estudiantes presentan la misma dificultad de la representación gráfica de los números naturales y los números enteros, ya que, los dos conjuntos numéricos los representan en una recta numérica, sin establecer la diferencia desde las representaciones gráficas que se propone en los libros de texto y de la historia de la matemática, donde los números naturales tiene como modelo "natural" de representación una semirrecta y los números enteros una recta.

5. En la entrevista de los docentes $\mathrm{D}_{11}$ y $\mathrm{D}_{21}$, se evidenció que estos no revelan una conciencia semiótica sobre la representación auxiliar de los conjuntos numéricos y la representación gráfica como representaciones de conjuntos infinitos. 


\section{Conclusiones}

La caracterización de las manifestaciones de la conciencia semiótica producidas en el docente, cuando se le problematiza su elección de representaciones semióticas en el proceso de enseñanza - aprendizaje de los conjuntos infinitos, evidencian la falta de conciencia semiótica de los docentes $\mathrm{D}_{11}$ y $\mathrm{D}_{21}$, así:

1. El docente $\mathrm{D}_{11}$ y la docente $\mathrm{D}_{21}$ eligieron para su cátedra una representación auxiliar de conjunto que presenta problemas semióticos. El docente $\mathrm{D}_{11}$ afirma que las "representaciones de sus estudiantes presenta errores y dificultades, debido a que ponen en el mismo conjunto los números racionales e irracionales, ellos no se dan cuenta de la diferencia entre el conjunto de los números racionales $(\mathbb{Q})$ y de los números irracionales (Q) '); la docente $\mathrm{D}_{21}$ declara [246]: "No sé, ya me confundí" al presentarle el problema semiótico que ella y sus estudiantes mostraron sobre la imagen de la representación auxiliar del conjunto de los números reales y que forma en el estudiante un conflicto cognitivo (D'Amore, 1999/2006).

Estas representaciones auxiliares elegidas por los docentes y construidas por los estudiantes, no están representando racionalmente el conjunto de los números reales y los conjuntos infinitos.

2. El docente $D_{11}$ y la docente $D_{21}$ eligieron para su cátedra una representación gráfica que presenta problemas semióticos. El docente $\mathrm{D}_{11}$ presenta problemas con sus estudiantes en la conversión entre la representación gráfica en la recta y la representación simbólica, ya que sus estudiantes no pueden representar gráficamente en la recta los números racionales negativos; la docente $\mathrm{D}_{21}$ propone que no es necesario poner la flecha, los puntos suspensivos y el símbolo de infinito $(\infty)$ en la recta numérica, ya que "para representar lo mismo, lo que pienso es que uno es suficiente", generando problemas en sus estudiantes al comprender esta representación gráfica de la recta numérica en los libros de texto; además, la docente y sus estudiantes representan en la misma recta numérica los números naturales y los números enteros, sin establecer la diferencia desde las representaciones gráficas que se propone en los libros de texto y de la historia de la matemática, donde los números naturales tiene como modelo "natural" de representación una semirrecta y los números enteros una recta. 
Estas representaciones gráficas elegidas por los docentes y construidas por los estudiantes, no están representando racionalmente el conjunto de los números reales y los conjuntos infinitos.

3. El docente $D_{11}$ y la docente $D_{21}$ no presentan una conciencia semiótica de las representaciones de los conjuntos infinitos. Además, al definir los conjuntos infinitos lo relacionan con la interpretación de un infinito potencial, definición que no permite la construcción cognitiva de los conjuntos infinitos dado que se necesita de un infinito actual.

A partir de estas evidencias, se debe generar un cambio en la conciencia semiótica de los docentes de matemáticas sobre la elección de las representaciones utilizadas en la construcción cognitiva y enseñanza de los conjuntos infinitos. Por último, se debe destacar que estas problemáticas son novedosas en la literatura de didáctica de la matemática.

Este artículo aporta, por una parte, datos empíricos que permiten un conocimiento consciente del uso de las representaciones semióticas, en la construcción cognitiva de los conjuntos infinitos. Y, por otra parte, contribuye en la línea de investigación clásica relativa a la didáctica del infinito y específicamente del aprendizaje de los conjuntos infinitos.

\section{Referencias}

Arrigo, G., \& D'Amore, B. (1999). "Lo veo, pero no lo creo”. Obstáculos epistemológicos y didácticos para la comprensión del infinito actual. Educación matemática, 11(1), 5-24.

Arrigo, G., \& D'Amore, B. (2002). "Lo vedo ma non ci credo...", secunda parte. Ancora su ostacoli epistemologic e didattici al proceso di comprensione di alcuni teorema di Georg Cantor. La matemática e la sua didattica, 10(1), 4-57.

Arrigo, G., D'Amore, B. y Sbaragli, S. (2011). Infiniti infiniti. Trento, Italia: Erickson. [Versión en idioma español: (2011). Infinitos infinitos. Bogotá, Colombia: Magisterio].

Becerra Galindo, H. M. (2017). Las problemáticas semióticas en las representaciones de los conjuntos infinitos en la práctica docente. La matematica e la sua didattica, 25(2), 191201.

Becerra Galindo, H. M. (2018). Las problemáticas semióticas en las representaciones de los conjuntos infinitos. II congreso de educación matemática de américa central y el caribe [II CEMACYC], 1-8.

Becerra Galindo, H. M., \& Font, V. (2019). Las problemáticas semióticas y la metáfora en las representaciones de los conjuntos infinitos. Revista Acta latinoamericana de matemática educativa [ALME], 32(1), 531-540. 
Brosseau, G. (1983). Ostacles Epistemologiques en Mathématiques. Recherches en didactique des mathématiques, 4(2), 165-198.

D'Amore, B. (1996). El infinito: una historia de conflictos, de sorpresas, de dudas. Epsilon, 36(1), 341-360.

D'Amore, B. (1999). Elementi di Didattica della Matematica. Bologna: Pitagora. Primer Premio Absoluto "Lo Stilo d Oro", secion Didactica, X Edicion del Premio Nacionale de Pedagogia Pescara. [Versión en idioma español: 2006, Bogotá: Magisterio].

D'Amore, B. (2002). La complejidad de la noética en matemáticas como causa de la falta de devolución. TED. Bogotá, Universidad Pedagógica Nacional, 11, 63-71.

D'Amore, B. (2011). La didáctica del infinito matemático. Sunto della Conferenza generale tenuta il 9 settembre 2011 al XXIV Coloquio Distrital de Matemáticas y Estadística, promosso dalle Universidad Distrital, Nacional y Pedagógica de Bogotá. In: AA. VV. (2011). Memorias del XXIV Coloquio Distrital de Matemáticas y Estadística, Bogotá, 8-10 septiembre 2011. CD. ISBN: 978-958-57050-0-5, 21-29.

D'Amore, B., Fandiño, Pinilla M. I., \& Iori, M. (2013). La semiótica en la didáctica de la matemática. Bogotá: Magisterio.

Dueñas, W., Garavito, A., \& Lara, G. (2007). Aciertos matemáticos 8. Bogotá, Colombia: Grupo Editorial Educar.

Duval, R. (1983). L'obstacle du dédoublement des objects mathématiques. Educational Studies in Mathematics, 14(4), 385-414.

Duval, R. (1993). Registres de Répresentation sémiotiques et fonctionnement cognitif de la Pensée. Annales de didactique et de sciences cognitive, 6(5), 37-65.

Duval, R. (1995). Sémiosis et pensé humaine. Registres sémiotiques et apprentissages intellectuels. Berne: Peter Lang. [Versión en idioma español: 1999, Cali: Universidad del Valle].

Duval, R. (2004). Los problemas fundamentales en el aprendizaje de las matemáticas y las formas superiores del desarrollo cognitivo (M. Vega, Trad.). Cali, Colombia: Universidad del Valle.

Duval, R. (2006). A cognitive analysis of problems of comprehension in a learning of mathematics. Educational Studies in Mathematics, 61(1-2), 103-131.

Duval R. (2008). Eight problems for a semiotic approach in mathematics education. En: Radford L., Schubring G., Seeger E. (Eds) (2008). Semiotics in mathematics education: epistemology, history, classroom, and culture. Rotterdam: Sense Publishers. 39-61.

Duval, R. (2017). Understanding the mathematical way of thinking-the registers of semiotic representations. Switzerland: Springer.

Duval, R., \& Sáenz-Ludlow, A. (2016). Comprensión y aprendizaje en matemáticas: perspectivas semióticas seleccionadas (M. Acosta, P. Perry. trad.). Bogotá, Colombia: Universidad Distrital Francisco José de Caldas.

Fandiño Pinilla M. I. (2010). Múltiples aspectos del aprendizaje de la matemática. Bogotá, Colombia: Magisterio. 
Fishbein, E., Tirosch, D., \& Hess, P. (1979). “The intuitions of infinity”. Educational Studies in Mathematics, 10(1), 3-40.

Iori, M. (2014). Matemática y semiótica en el aula: un punto de vista necesario. In C. J. Mosquera Suárez (Ed.), Miradas contemporáneas en educación: Algunos puntos clave para el debate (pp. 27-44). Chía (Colombia): Universidad Distrital Francisco José de Caldas.

Moreno, L., \& Walddeg, G. (1991). The conceptual evolution of actual mathematical infinity. Education Studies in Mathematics, 22(3), 211-231.

Moreno, J., Roldán, D., \& Romero, F. (2011). Norma Matemáticas para pensar 11. Bogotá, Colombia: Carvajal Educación S.A.S.

Pino-Fan, L., Guzmán, I., Duval, R., \& Font, V. (2015). The theory of registers of semiotic representation and the onto-semiotic approach to mathematical cognition and instruction: linking looks for the study of mathematical understanding. In Beswick, K.., Muir, T., \& Wells, J. (Eds.). Proceedings of 39th Psychology of Mathematics Education conference, Vol. 4, pp. 33-40. Hobart, Australia: PME.

Tsamir, P. (2000). La comprensione dell'infinito attuale nei futuri insegnanti. La matemática e la sua didattica, 8(2), 167-207.

Autor:

Héctor Mauricio Becerra Galindo. https://orcid.org/0000-0002-5477-4680; Universidad Distrital Francisco José de Caldas; Colombia. Candidato a Doctor en Educación, con énfasis en Educación Matemática del Doctorado Interinstitucional en Educación (DIE) de la Universidad Distrital Francisco José de Caldas. Magister en Educación, con énfasis en Educación Matemática de la Universidad de los Andes (2012). Licenciado en Educación Básica con Énfasis en Matemáticas de la Universidad Distrital Francisco José de Caldas (2006). Integrante del grupo de investigación MESCUD (Matemáticas Escolares Universidad Distrital, Bogotá, Colombia) y NRD (Nucleo di Ricerca in Didattica della Matematica, Universidad de Bologna, Italia). Docente de matemática de la Secretaria de Educación del Distrito (SED), Bogotá, Colombia. 